\title{
Efficacy and Toxicity of Pembrolizumab in Pediatric Metastatic Recurrent Melanoma
}

\author{
AGATA MARJANSKA ${ }^{1}$, PRZEMYSLAW GALAZKA ${ }^{2}$, MICHAL MARJANSKI ${ }^{3}$, \\ MARIUSZ WYSOCKI ${ }^{1}$ and JAN STYCZYNSKI ${ }^{1}$ \\ ${ }^{1}$ Department of Pediatric Hematology and Oncology, Nicolaus Copernicus University Torun, \\ Jurasz University Hospital, Collegium Medicum, Bydgoszcz, Poland; \\ ${ }^{2}$ Department of General and Oncological Surgery for Children and Adolescents, \\ Nicolaus Copernicus University Torun, Jurasz University Hospital, Collegium Medicum, Bydgoszcz, Poland; \\ ${ }^{3}$ Clinical Oncology Department, Oncology Center, Bydgoszcz, Poland
}

\begin{abstract}
Background/Aim: Malignant melanoma is a rare disease in the pediatric population and there are no recommendations regarding its management in children, while the current standard of care in metastatic or unresectable melanoma in adult patients includes immunotherapy (anti-CTLA-4 and anti-PD-1 antibodies). Advances in the management of adults with melanoma offer the prospect of promising therapeutic options for children. Case Report: We describe a case of a 7-year-old patient with recurrent metastatic melanoma, for whom pembrolizumab was used as an adjuvant therapy on compassionate use basis. Conclusion: Due to adverse events, the treatment was discontinued after 5 months of pembrolizumab, but with 12 months of follow-up, patient remains in complete remission.
\end{abstract}

Malignant melanoma is a very rare disease in children. Less than $1 \%$ of all melanoma cases occur in prepubertal patients. Conflicting data exist on the outcomes of pediatric melanoma compared to older patients. More recent analyses suggest a better outcome in children when compared with adults, what implies that children might have a different biologic form of malignant melanoma than adults (1).

Nowadays the standard of care in metastatic or unresectable melanoma in adult patients include immunotherapy with anticytotoxic T-lymphocyte-associated protein 4 (CTLA-4) and anti-programmed cell death protein 1 (PD-1) antibodies, which is an unprecedented breakthrough in the treatment of this

Correspondence to: Agata Marjanska, MD, Department of Pediatric Hematology and Oncology, Collegium Medicum, Nicolaus Copernicus University Torun, Antoni Jurasz University Hospital No. 1, ul. Sklodowskiej-Curie 9, 85-094 Bydgoszcz, Poland. Tel: +48 525854860, Fax: +48 525854087, e-mail: agata.marjanska@wp.pl

Key Words: Pembrolizumab, malignant melanoma, immunotherapy. malignancy and has contributed to significantly improved prognosis. However, an access to modern therapeutic options in children is currently limited. Recently, the only new immune medication registered for the use in children aged over 12 years is ipilimumab, an anti-CTLA-4 antibody. Other immunotherapeutics, such as anti-PD-1 inhibitors pembrolizumab and nivolumab or targeted therapies, are registered only for adult patients $(2,3)$.

With the rarity of melanoma in children and the lack of clinical trials, there are no recommendations on management in this malignancy in pediatric patients. In exceptional cases, the ultimate therapy can be the use of off-label drugs. In this report we present a case of a child with metastatic recurrent melanoma, for whom pembrolizumab was successfully applied as a method of treatment.

\section{Case Report}

A 4-year-old female patient was diagnosed for malignant melanoma stage IIIC by TNM classification, while carrying no $B R A F$ mutation, with primary tumor localized on right arm and presence of metastases to axillary lymph nodes. Total surgical resection with wide margin of the primary tumor and removal of the axillary node package was performed followed by adjuvant therapy with interferon $\alpha$ 2B (IFN $\alpha-2 B$ ). The patient received IFN $\alpha-2 B$ over one year in two phases: induction $\left(20\right.$ million Units $/ \mathrm{m}^{2}$ i.v., 5 days/week for 4 weeks) and maintenance $(10$ million Units $/ \mathrm{m}^{2}$ s.c. 3 times/week for 48 weeks). No adverse events (AEs) were observed during treatment with IFN $\alpha-2 \mathrm{~B}$. After the end of the treatment, complete response (CR) persisted for two years.

Two months after the end of the IFN $\alpha-2 B$ therapy, autoimmune choroidal inflammation of the right eye was diagnosed in the patient. Topical steroids were sufficient to keep the diseases stable without exacerbation. 
At the age of 7 years, the patient was diagnosed for recurrent melanoma with two foci localized in the soft tissue of the right forearm and right thigh (Figure 1). Both changes were surgically removed, histologically confirmed (BRAFnegative) and re-treatment with IFN $\alpha$-2B was applied.

Two months later, PET scan was performed, and the presence of new lesions suspected of metastases within the soft tissues of the right thigh were revealed. Metastases were surgically resected, followed by adjuvant therapy with pembrolizumab, which was implemented on compassionate use basis. The drug was applied intravenously at dose of 2 $\mathrm{mg} / \mathrm{kg}$ every 3 weeks. Based on PET imaging after the third dose of the drug, CR was achieved.

After the fourth dose of pembrolizumab, a decreased vision on the right eye with exacerbation of uveitis and pain in the left knee and left foot were observed. Thus, systemic oligoarticular type of juvenile idiopathic arthritis (JIA) was diagnosed. With the administration of steroids and temporary discontinuation of pembrolizumab, remission of the articular symptoms and stabilization of uveitis was achieved.

After four weeks break, the patient received three next courses of pembrolizumab simultaneously with low-dose steroids. However, recurrence of arthritis and significant exacerbation of uveitis has developed, which this time was not controlled by increasing doses of steroids. Due to development of Cushing syndrome, significant deterioration of the quality of life and the risk of blindness in the right eye, a decision about cessation of the treatment with pembrolizumab was made. Patient received seven doses of pembrolizumab in total, with CR of melanoma symptoms confirmed by PET scan. Currently, with 12-months of follow-up after discontinuation of the therapy, a CR is observed both clinically and in MRI imaging. Symptoms of JIA have been managed with steroids and methotrexate for 4 months after the end of the immunotherapy with complete resolution.

\section{Discussion}

According to NCCN Guidelines for malignant melanoma in adult patients, the adjuvant immunotherapy should be considered in all cases with distant metastatic disease after primary treatment (4). The use of adjuvant high-dose interferon in adult and pediatric patients with high-risk melanoma is controversial. Those who dispute the use of adjuvant interferon cite modest benefit at the expense of cost and toxicity as arguments against its use (5). Currently, there are three immunomodulating agents available for melanoma, which were shown to improve overall survival in patients with metastatic melanoma without BRAF mutation: pembrolizumab, nivolumab and ipilimumab $(2,6)$.

There exist no data in the literature on the use of anti-PD1 or anti-CTLA-4 in pediatric melanoma. In our patient, after

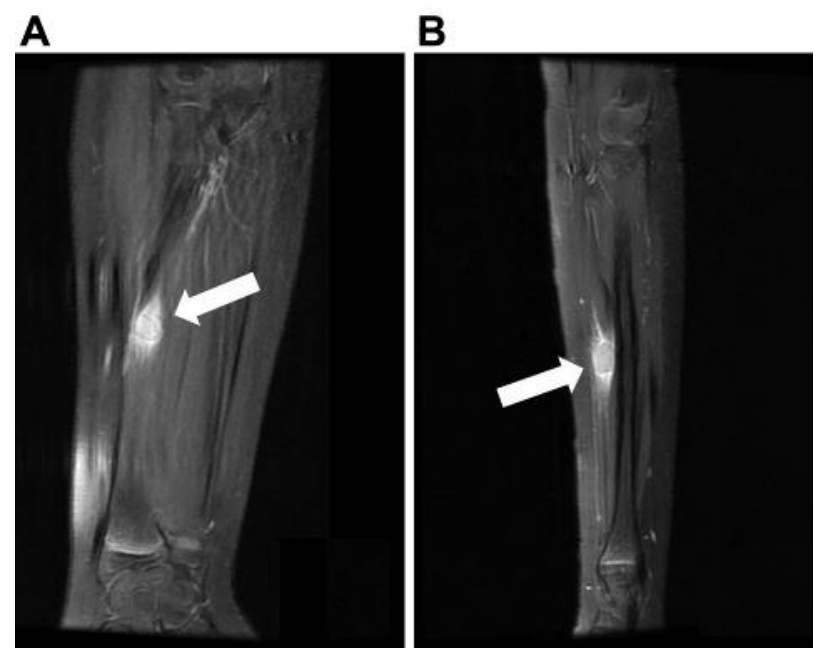

Figure 1. Melanoma metastases in the soft tissues of the (A) thigh and (B) forearm, visualized in the MRI.

the second progression, and dismal effect of IFN, pembrolizumab was applied on compassionate use basis. However, due to immune-related adverse events, the treatment was discontinued after 5 months of therapy. Nevertheless, with 12-months of follow-up, the child is free from progression. The immunotherapy was stopped because of adverse events occurring after 7 th dose of pembrolizumab. Withdrawal of immunotherapy and careful management of JIA were necessary for the alleviation of symptoms resulting of adverse events.

Pembrolizumab is an anti-PD1 monoclonal antibody, approved for treatment of metastatic melanoma in adults. Pembrolizumab mediates its antitumour effect by unleashing latent tumor-specific T-cell responses. Chronic PD-1 blockade may be marked by immune dysregulation, which can manifest in a diverse range of organ-related toxicities. The incidence of these immune-related adverse events (irAEs) tends to increase slowly with continued exposure to anti-PD1 therapy. Most irAEs related to PD1 blockade appear during the first 6 months of treatment $(7,8)$. In one study, the incidence of AEs involving the use of pembrolizumab was $12 \%$. These patients experienced grade III- $\mathrm{V}^{\circ}$ of drug-related AEs. The most common AEs were pneumonitis, rash, pruritus, endocrinopathies, hepatitis, gastrointestinal diarrhea and colitis. Fatigue was often experienced among patients receiving therapy with pembrolizumab. The arthritis and uveitis (any grades) are described in $5.2 \%$ and $<1 \%$ patients, respectively, after treatment with pembrolizumab (9-12). Most irAEs occur during the first 2 to 6 months of treatment, however, these can be effectively managed with the use of corticosteroid treatments (13).

Robert et al. described long-term follow-up in patients with metastatic melanoma treated with pembrolizumab, 
where 19 patients discontinued treatment before second course of immunotherapy because of AEs or per investigator decision. Among these patients $78.9 \%$ remained in $\mathrm{CR}$ at data cutoff (14). Therefore, discontinuation of the therapy after 7 th cycle of pembrolizumab in our patient, will not be equivalent to the lack of effectiveness of the treatment.

In conclusion, the 5-months use of pembrolizumab in a child with advanced melanoma was effective. The child stays in CR after two previous relapses of disseminated melanoma. The side-effects observed after drug administration were manageable.

\section{Conflicts of Interest}

None of the Authors have any conflicts of interest to disclose.

\section{Authors' Contributions}

Design of the study: AM, MW, JS. Collection and analysis of data: AM, PG, MM. Writing draft: AM. Critical revision: JS. Final approval of the manuscript: all authors.

\section{Acknowledgements}

The Authors thank the nurses from the department for their perfect care of patient, and radiologists for their excellent service.

\section{References}

1 Moore-Olufemi S, Herzog C, Warneke C, Gershenwald JE, Mansfield P, Ross M, Prieto V, Lally KP and Hayes-Jordan A: Outcomes in pediatric melanoma: Comparing prepubertal to adolescent pediatric patients. Ann Surg 253(6): 1211-1215, 2011. PMID: 21451389. DOI: 10.1097/SLA.0b013e318217e852

2 Lugowska I, Teterycz P and Rutkowski P: Immunotherapy of melanoma. Contemp Oncol (Pozn) 22(1A): 61-67, 2018. PMID: 29628796. DOI: $10.5114 /$ wo.2018.73889

3 Mattila K, Raanta P, Lahtela V, Pyrhonen S, Koskivuo I and Vihinen P: Long-term survival of stage iv melanoma patients treated with bold combination chemotherapy and intermediatedose subcutaneous interferon-alpha. Anticancer Res 38(11): 63936397, 2018. PMID: 30396963. DOI: 10.21873/anticanres.12999

4 Coit DG, Thompson JA, Algazi A, Andtbacka R, Bichakjian CK, Carson WE, 3rd, Daniels GA, DiMaio D, Ernstoff M, Fields RC, Fleming MD, Gonzalez R, Guild V, Halpern AC, Hodi FS, Jr., Joseph RW, Lange JR, Martini MC, Materin MA, Olszanski AJ, Ross MI, Salama AK, Skitzki J, Sosman J, Swetter SM, Tanabe KK, Torres-Roca JF, Trisal V, Urist MM, McMillian N and Engh A: Melanoma, version 2.2016, NCCN clinical practice guidelines in oncology. J Natl Compr Canc Netw 14(4): 450473, 2016. PMID: 27059193.
5 Navid F, Herzog CE, Sandoval J, Daryani VM, Stewart CF, Gattuso J, Mandrell B, Phipps S, Chemaitilly W, Sykes A, Davidoff AM, Shulkin BL, Bahrami A, Furman WL, Mao S, Wu J, Schiff D, Rao B and Pappo A: Feasibility of pegylated interferon in children and young adults with resected high-risk melanoma. Pediatr Blood Cancer 63(7): 1207-1213, 2016. PMID: 27038395. DOI: $10.1002 / \mathrm{pbc} .25983$

6 Mazzio EA and Soliman KFA: Whole-transcriptomic profile of SK-MEL-3 melanoma cells treated with the histone deacetylase inhibitor: Trichostatin A. Cancer Genomics Proteomics 15(5): 349-364, 2018. PMID: 30194076. DOI: $10.21873 / \mathrm{cgp} .20094$

7 Hsieh AH, Faithfull S and Brown MP: Risk of cumulative toxicity after complete melanoma response with pembrolizumab. BMJ Case Rep 2017, 2017. PMID: 28148549. DOI: 10.1136/ bcr-2016-218308

8 Sukari A, Nagasaka M, Alhasan R, Patel D, Wozniak A, Ramchandren R, Vaishampayan U, Weise A, Flaherty L, Jang H, Kim S and Gadgeel S: Cancer site and adverse events induced by immune checkpoint inhibitors: A retrospective analysis of real-life experience at a single institution. Anticancer Res 39(2): 781-790, 2019. PMID: 30711957. DOI: 10.21873/anticanres.13175

9 Spain L, Younger E, Hatipoglu E and Larkin J: Pembrolizumab in the management of metastatic melanoma. Melanoma Manag 2(4): 315-325, 2015. PMID: 30190860. DOI: 10.2217/mmt. 15.33

10 So AC and Board RE: Real-world experience with pembrolizumab toxicities in advanced melanoma patients: A single-center experience in the UK. Melanoma Manag 5(1): MMT05, 2018. PMID: 30190931. DOI: 10.2217/mmt-2017-0028

11 Ariyasu H, Inaba H, Ota T, Yamaoka H, Furukawa Y, Iwakura H, Doi N, Yamamoto Y and Akamizu T: Thyrotoxicosis and adrenocortical hormone deficiency during immune-checkpoint inhibitor treatment for malignant melanoma. In Vivo 32(2): 345351, 2018. PMID: 29475919. DOI: 10.21873/invivo.11244

12 Yamazaki H, Iwasaki H, Yamashita T, Yoshida T, Suganuma N, Yamanaka T, Masudo K, Nakayama H, Kohagura K, Rino Y and Masuda M: Potential risk factors for nivolumab-induced thyroid dysfunction. In Vivo 31(6): 1225-1228, 2017. PMID: 29102951. DOI: 10.21873 /invivo.11195

13 Jazirehi AR, Lim A and Dinh T: Pd-1 inhibition and treatment of advanced melanoma-role of pembrolizumab. Am J Cancer Res 6(10): 2117-2128, 2016. PMID: 27822406.

14 Robert C, Ribas A, Hamid O, Daud A, Wolchok JD, Joshua AM, Hwu WJ, Weber JS, Gangadhar TC, Joseph RW, Dronca R, Patnaik A, Zarour H, Kefford R, Hersey P, Zhang J, Anderson J, Diede SJ, Ebbinghaus S and Hodi FS: Durable complete response after discontinuation of pembrolizumab in patients with metastatic melanoma. J Clin Oncol 36(17): 1668-1674, 2018. PMID: 29283791. DOI: 10.1200/JCO.2017.75.6270

Received April 12, 2019

Revised May 28, 2019

Accepted June 3, 2019 